\title{
Biopsy of lung lesions under CT control
}

\author{
Miloslav Marela ${ }^{a}$, Radek Padr ${ }^{\mathrm{b}}$, Libor Fila ${ }^{\mathrm{a}}$, Dmitry Rakitaa ${ }^{\mathrm{a}}$, Fernando Casas Mendez ${ }^{\mathrm{a}}$, Linda Capkovac, Vaclav Capek ${ }^{\mathrm{d}}$, \\ Radim Pavlik
}

\begin{abstract}
Objectives. With the increasing number of detected lung nodules and the need for morphological verification, the number of CT- controlled biopsies is increasing. The aim of this study was to assess the risks and benefits of these biopsies.
\end{abstract}

Methods. This is a prospective and observational study. We evaluated 101 punctures performed on a group of 90 consecutive patients in the Department of Radiology.

Results. In patients with a mean age of 66 years, with mostly accidentally detected lung nodules, we observed complications 38 times. The most common were minor pneumothoraxes or insignificant bleedings. In 6 patients, the complications were more serious, 5 times the pneumothoraxes required chest drainage, once massive hemoptysis was recorded. The lesions were successfully biopsied 78 times, the target was missed 23 times. The diagnosis of lung cancer (LC) was confirmed in 60 patients, 49 LCs were verified by puncture under CT control. 42\% (25/60) of patients with LC were diagnosed in TNM stages I and II. 23\% (14/60) of patients with LC were treated surgically. The remaining 30 patients most often suffered from lung metastazes (13/30), in 8 of them an inflammatory lung disease was diagnosed. 69 patients underwent bronchoscopy, in only 19\% (13/69) it contributed to the diagnosis. In a model "screening like" group of 49 patients with only randomly detected lung deposits, we diagnosed LC in 76\% (37/49). 49\% (18/37) were in TNM stage I and II, 11 were treated surgically.

Conclusions. CT-controlled biopsy of lung lesions is an effective and safe diagnostic method.

Key words: CT-controlled biopsy, lung nodules, lung cancer

Received: May 26, 2020; Revised: September 4, 2020; Accepted: September 8, 2020; Available online: September 17, 2020 https://doi.org/10.5507/bp.2020.040

(c) 2021 The Authors; https://creativecommons.org/licenses/by/4.0/

${ }^{a}$ Department of Pulmonology, University Hospital Motol and 2nd Faculty of Medicine, Charles University, Prague, Czech Republic ${ }^{b}$ Department of Radiology, University Hospital Motol, and 2nd Faculty of Medicine, Charles University, Prague, Czech Republic 'Department of Pathology and Molecular Medicine, University Hospital Motol, and 2nd Faculty of Medicine, Charles University, Prague, Czech Republic

${ }^{d}$ Bioinformatics Centre, University Hospital Motol, and 2nd Faculty of Medicine, Charles University, Prague, Czech Republic Corresponding author: Miloslav Marel, e-mail: miloslav.marel@fnmotol.cz

\section{INTRODUCTION}

In addition to purely pneumological methods (bronchoscopy, EBUS-guided biopsy of lung nodules using endobronchial ultrasound), newer methods performed by interventional radiologists are increasingly being used in the diagnosis of lung lesions ${ }^{1-4}$. Their importance will increase with the growing number of early diagnosed lung nodules. We have observed this increase in clinical practice quite intensively in recent years. This is a consequence of easier availability of computer tomography (CT) examinations (not only in regional or faculty centers) and its more frequent indications. A further increase of diagnosed lung nodules can be expected shortly after the start of low dose spiral CT screening for lung cancer ${ }^{5}$.

Therefore, we decided to examine what benefits we can expect from punctures of pulmonary nodules under CT control for their diagnosis and what their complications are. At the same time, we wanted to assess the effectiveness of bronchoscopy for the diagnosis of these lesions and on a model set of "screening like" pulmonary lesions determine what diagnostic benefit can be expected from different methods and what treatment will be indicated.

\section{METHODS}

From January 2018 to June 2019, we prospectively collected data from all consecutive patients who underwent lung biopsy under CT control at the Department of Radiology in University Hospital Motol, 2nd Medical School, Charles University, Prague. Radiologists used Tru-Cut 14 biopsy needles (widest for subpleural lesions) and 16 to 18 biopsy needles (thinnest for deep lung lesions). Subsequently, we monitored the diagnostic process, including bronchoscopy, cytological and histological findings and other examination methods, until the final diagnosis and treatment were determined. First, we summarized the results from the point of view of radiologists, for whom it may be beneficial to know the risks and effectiveness of each individual puncture, of which 101 were accomplished. Subsequently, we evaluated the benefit of lung nodules diagnosis using transparietal biopsy in terms 
of individual patients, of which there were 90. Rebiopsy was undergone by 7 patients, re-rebiopsy 2 patients. At the same time, we evaluated the possibilities of bronchoscopic (BRS) verification of lung lesions in patients in our group who underwent this examination. Finally, we tried to create a model set of patients with lung nodules detected more or less randomly, as they would be diagnosed in an asymptomatic at-risk population participating in a planned low-dose CT lung cancer screening. The results were evaluated statistically using the logistic regression test (Spearman's correlation coefficient) and the relationship between the quantities test.

\section{RESULTS}

We performed 101 punctures in 90 patients (7x two in one patient (pts), $2 x$ three in one pts). There were 29 women, 61 men. The oldest patient was 82 years old, the youngest 25 years old, the average age of the group was 65 years.

Pulmonary nodules were detected in our group of 90 patients by a random chest X-ray 4 times, the symptoms were the reason for radiological examination 29 times and 57 nodules were found during X-ray or CT examination indicated for the control of other diseases. The lesions were biopsied in the right lung 46 times, in the left lung 52 times, in the pleura twice and in the chest wall once. The most common nodules were in lung segment B6 1.dx and B10 1.dx (11x) and in B6 1.sin and B10 1.sin (12x). According to the longest axis of nodules, we divided the set into 4 groups: $8-29 \mathrm{~mm}$ "small" $(\mathrm{n}=48), 30-49 \mathrm{~mm}$ "medium" ( $n=21), 50-69 \mathrm{~mm}$ "large" $(\mathrm{n}=17)$ and 70-300 mm "giant" $(n=15)$. The average size of the longest axis was $44 \mathrm{~mm}$.

Complications occurred in 38/101 (38\%) punctures. The cause was either spontaneously receding small pneumothorax (PNO) or minor bleeding into lung parenchyma. Only in 6/90 patients (7\%) the complications of a lung lesion biopsy required treatment. PNO was drained 5 times after puncture, massive hemoptysis was solved once, managed by intubation and several days of intensive care.

The statistical test (logistic regression) showed a significant association between distance of the center of the nodules from the chest wall and the occurrence of complications $(P=0.0113)$. The further the lesion was from the thoracic wall, i.e. the more centrally located in the lung, the higher was the risk of complications (Fig. 1). Statistically (logistic regression), we further showed that the smaller the nodules, the greater the risk of complications $(P=0.0007)$ (Fig. 2).

Interventional radiologists were successful in biopsying the lesion in 78/101 punctures (77\%), 23 times the biopsy needle missed the lesion. The success of the impact of the nodules was only marginally related to its size, the test of relationships did not reach statistical significance $(P=0.0724)$ (Fig. 3).

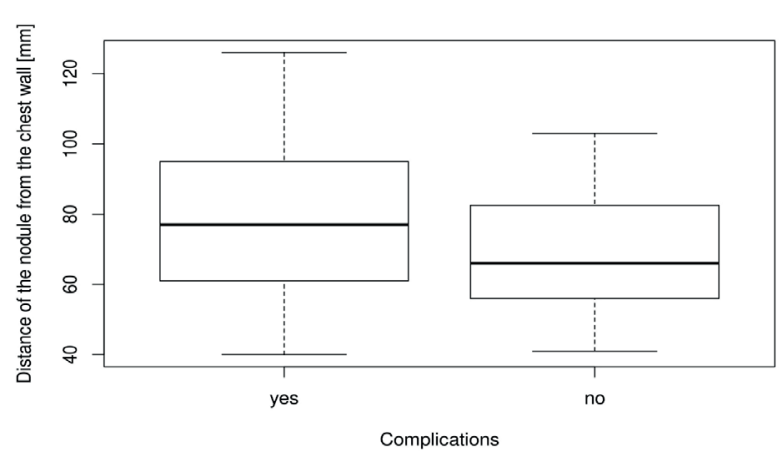

Fig. 1. The relationship of the distance of the centre of nodules from the chest wall and the occurrence of complications (logistic regression effect test, $P=0.0113$ )

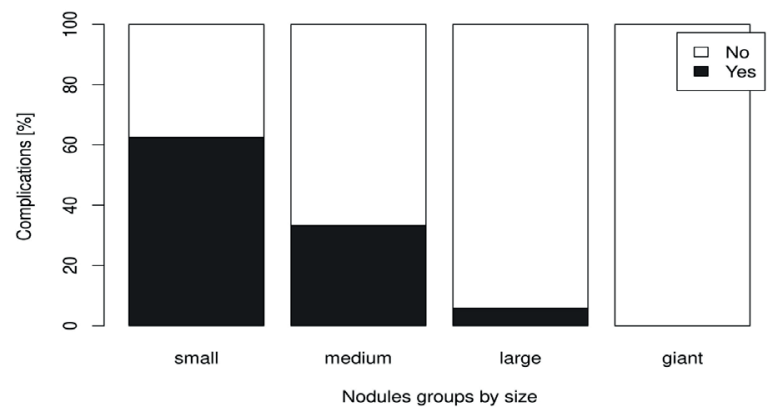

Fig. 2. Frequency of puncture complications in nodule groups by size (logistic regression effect test, $P=0.0007$ )

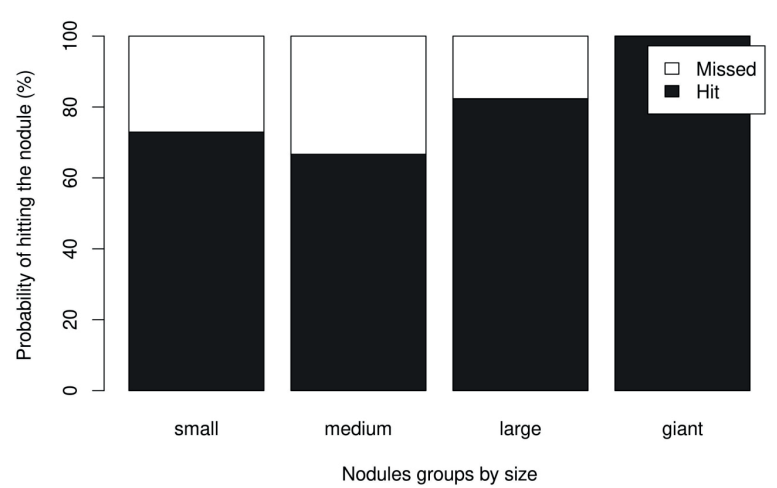

Fig. 3. Success of biopsy according to nodule groups by size (test of connection between quantities, $P=0.0724$ ).

We divided the obtained tissue samples into representative (suitable to set histologic diagnosis) and unrepresentative (not suitable). A representative tissue sample was obtained in 74 punctures, a non-representative one in the remaining 27 punctures. In 23 cases, tissue outside the lesion was sampled, in 4 cases the tissue was removed from the lesion, but the material was insufficient to set the precise diagnosis (necrotic tissue $2 \mathrm{x}$, connective tissue stroma, unspecified inflammation). In 71 patients with representative biopsy tissue sample, the tru-cut biopsy diagnosis correlated with the definitive specimen 
diagnosis, in 3 remaining cases, the definitive diagnosis was distinct. We evaluated the localisation of most of the non-representative samples. Segments B1 and B9 in the right lung, and B6 in the left lung can be considered as risk localities with the highest incidence of inaccuracies ( $3 \times$ higher incidence than that of representative samples).

We also assessed the ability of an experienced radiologist to estimate the etiology of the lesion according to the $\mathrm{CT}$ finding. The radiological character of the lesion corresponded to lung carcinoma in 34 cases. Concordance with the final diagnosis was in 25 lesions (74\%). Radiologists have been less successful in assessing the radiological nature of metastases. 19 foci had this character, but the final diagnosis confirmed that only $6(31 \%)$ of them were metastases histologically.

We compared also the CT density of nodules in Hounsfield units with the final diagnosis. There were no statistically significant differences (Kruskal-Wallis test, $P=0.5356$ ) (Fig.4).

When evaluating the representativeness of the samples (including rebiopsies), a representative sample was obtained in 74 patients ( 8 of them had rebiopsies), unrepresentative in the remaining 16 , while in one patient neither rebiopsy yielded suitable diagnostic material.

The diagnosis of lung cancer was confirmed in 60 patients in our group, 49 (82\%) LCs were verified by biopsy under CT control, 11 LCs were diagnosed by another method. 6 of them were verified during surgery, 5 were diagnosed by bronchoscopy.

The sensitivity of biopsy under CT control in the recognition of LC was $81.7 \%$ (proportion of 49 positive tests from all 60 patients), its specificity $96.7 \%$ (proportion of 29 negative tests from all 30 healthy patients). The positive predictive value (proportion of 49 patients from all 50 positive tests) was $98.0 \%$, the negative predictive value (proportion of 29 healthy from all 40 negative tests) was $72.5 \%$.

The stages of TNM in 49 lung cancers diagnosed by transparietal lung biopsy and in 11 LC diagnosed by other methods are specified in Table 1. In the first group, there were 29 adenocarcinomas, 12 epidermoid LCs, 5 small cell LCs, one unspecified non-small cell LC, large cell LC

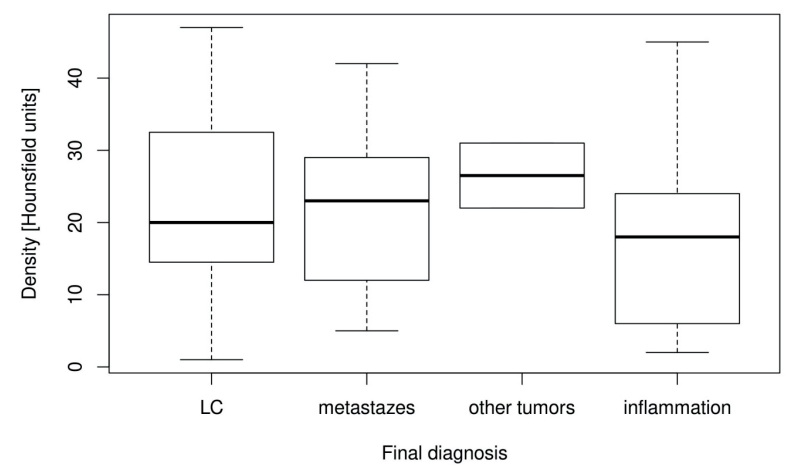

Fig. 4. CT density of pulmonary lesions (Hounsfield units) and final histologic diagnoses differences (Kruskal-Wallis test, $P=0.5356$ ) and one typical carcinoid. Of the LC-verified punctures, $8(16 \%)$ patients were operated on.

In the group of 11 LCs diagnosed by other methods there were 4 epidermoid LC, 4x adenocarcinomas, one small cell LC, unspecified non-small cell LC, and an atypical carcinoid.

Table 1. Numbers of patients with LC diagnosed by biopsy under CT control and other methods and their TNM stages.

\begin{tabular}{lcccc}
\hline TNM & $\begin{array}{c}\text { CT } \\
\text { biopsy }\end{array}$ & $\begin{array}{c}\text { Other diagnostic } \\
\text { methods }\end{array}$ & Total & $\%$ \\
\hline I & 6 & 5 & 11 & 18 \\
II & 12 & 2 & 14 & 24 \\
III & 10 & 2 & 12 & 20 \\
IV & 21 & 2 & 23 & 38 \\
Total & 49 & 11 & 60 & 100 \\
\hline
\end{tabular}

In the remaining 30 patients, 13 cases of metastases to the lungs (sarcoma, clear cell renal cell carcinoma, etc.), 6 of non-specific inflammation, 2 liposarcomas, hamartoma, granulomatosis with polyangiitis, pulmonary fibrosis, sarcoidosis, and 2 cases of tuberculosis were diagnosed. 3 patients moved and their final diagnosis remained unknown.

From the whole investigated cohort, 19 out of 90 patients were surgically treated. There were total of 14 LCs (9x adenocarcinoma, $2 \mathrm{x}$ epidermoid carcinoma, $1 \mathrm{x}$ non small cell LC not otherwise specified, 1x typical carcinoid, 1x atypical carcinoid). This represents 23\% (14/60) of all LCs diagnosed in our study. The remaining 5 surgical procedures comprised 2 cases of metastatic breast and renal cancer, 1 case of a benign hamartoma, solitary fibrous tumor and tuberculosis.

Additionally, bronchoscopy was performed in 69 patients, for various reasons it was not accomplished in 21 cases. These patients were sent for biopsy of the lesion under CT control mostly from non-pneumological departments. Bronchoscopic findings, as well as cytological and histological samples taken, were negative in 56 patients. Bronchoscopy was macroscopically, cytologically and / or histologically positive in 4, 8, 1 patients respectivelly, in total in 13 pts (19\%). Endobronchial ultrasound (EBUS) was used 10 times during these examinations, only in two cases the cytological sample was positive. In 16 BRS, radial EBUS was also used and contributed to the diagnosis of malignant lesion in 3 cases.

Finally, we generated a subset of cases from our file, which was called "screening-like" pulmonary lesions. We selected 49 patients from the entire cohort of 90 who were to some extent similar to the presumed screening population. We included patients whose lesions were found accidentally by a random chest X-ray or CT examination (CT of the abdomen with an accidental finding of a lesion in the lower lobe of the lung). We also included patients without symptoms who were monitored for chronic obstructive pulmonary disease (COPD) or other disorder. In $75 \%$ (37/49), the diagnosis of lung cancer was set. In 
21/37 cases it was an adenocarcinoma, in 9 cases epidermoid carcinoma, in 3 patients small cell lung carcinoma, and in 4 cases other type of LC. $49 \%$ (18/37) of these malignancies were diagnosed in early TNM stages (I and II), and 30\% (11/37) of patients underwent surgery. Lobectomy was performed by thoracic surgeons in $9 / 11$ cases, wedge resection in $2 / 11.26 / 37$ patients with LC were inoperable, 14 for the extent of the disease, 3 had small cell LC treated with chemotherapy. In 6 patients surgery was contraindicated for overall poor performance status and polymorbidity and 3 patients refused surgery entirely.

The mean age of patients with lung cancer in this "screening like" group was 71 years, there were only 9 patients under 65 years. The median size of the nodules was $20 \mathrm{~mm}$. 19 lung cancers occured in the right lung, 18 in the left lung. On the left side, LC was most frequently found in the upper lobe $(10 / 37)$, on the right side the lower lobe was most often affected (12/37). TNM stage of the "screening like" diagnosed lung cancers and their operability are listed in Table 2. The remaining 12 patients from the "screening like" group suffered from benign diseases such as inflammatory or fibrosing diseases or benign tumors (hamartoma). 4 of them were treated surgically.

Table 2. TNM stages of "screening like" diagnosed lung cancers and their operability.

\begin{tabular}{lcccr}
\hline $\begin{array}{l}\text { TNM } \\
\text { stages }\end{array}$ & $\begin{array}{c}\text { Number } \\
\text { of pts }\end{array}$ & $\%$ & $\begin{array}{c}\text { Underwent } \\
\text { surgery }\end{array}$ & $\%$ \\
\hline I & 10 & 27 & 8 & 22 \\
II & 8 & 22 & 2 & 5 \\
III & 6 & 16 & 1 & 3 \\
IV & 13 & 35 & 0 & 0 \\
total & 37 & 100 & 11 & 30 \\
\hline
\end{tabular}

\section{DISCUSSION}

The aim of this study was to assess as accurately as possible the benefits and risks of the diagnostic method often indicated, needle biopsy of lung deposits under CT control. Our effort was to contribute to the improvement of both our (pneumological) indications and the practice performed by radiologists. The set of 101 consecutive punctures in 90 patients was not large enough to allow us to draw any far-reaching conclusions. However, consequent to our results, we can expect minor complications in about a third of the procedures, major complications in about $6 \%$. Higher number of complications occurs with more centrally located lesions and a smaller size of lung nodules. This is in concordance with other studies ${ }^{6}$.

It can also be concluded that the biopsy needle yielded diagnostic material from the lesions in about $73 \%(74 / 101)$ of biopsies, which corresponds with other authors ${ }^{7}$. In a quarter of them, for various reasons, the procedure failed. In such cases, rebiopsy was the method of choice with success rate of $89 \%(8 / 9)$ in our sample of patients. Of interest is the fact that we did not findany statistically significant relationship between biopsy success rate and size of the nodules $(P=0.0724)$ in opposite to findings of Huang et al. ${ }^{6}$

According to our results, the success rate of an experienced radiologist concerning the etiology of the lesion was $74 \%$ in diagnosing lung cancer. In contrast, its etiology cannot be inferred from the density of the lesion (in Hounsfield units). We diagnosed lung cancer in 60 patients in our group. Biopsy under CT control was the fundamental diagnostic method in 49 patients, 11 were diagnosed by a different method. In general, it can be estimated that up to $2 / 3$ of lung nodules sent for biopsy under CT control are caused by LC and that about $3 / 4$ of them can be verified by this method. $42 \%$ were diagnosed in early TNM stages (I and II). In total, 23\% of patients with LC could be operated on.

69 patients underwent bronchoscopy, which was successful in diagnosing lung lesions in $19 \%$. This corresponds to the previously determined $20 \%$ of positive bronchoscopical findings in peripheral lung lesions in our group of patients from years 2004-2006 (ref. ${ }^{8}$ ).

New bronchological methods, such as EBUS or radial EBUS, have contributed only minimally to the verification of peripheral lesions in patients in our group. They were successful in 20\% (2/10) and 19\% (3/16) of cases respectively. According to the literature, we could expect a much higher yield of radial EBUS in the diagnosis of peripheral lung lesions. In a study by Hsia et al., radial EBUS was successful in 65\% verifying 40 lung deposits under $30 \mathrm{~mm}$ (ref. ${ }^{9}$ ). Our success rate was only $20 \%$. The cause could be possibly due to our inexperience with this method that was newly introduced to our department at the time of the study.

In our model-created "screening like" group of 49 patients, lung cancer was diagnosed in 75\% (37/49) of cases. The size of these lesions was approximately $20 \mathrm{~mm}$, most often the upper left and lower right lobes were affected. Less often than we expected (in 30\%), we indicated resection treatment in such diagnosed LCs, although $49 \%$ of them were diagnosed in early TNM stages (I. and II.). In comparison according to the Dante study ${ }^{10}, 65 \%(39 / 60)$ of LCs detected by screenings were surgically treated. In the US National Lung Screening Trial (NLST) it was $60 \%(642 / 1060)$ of the detected LCs (ref. $\left.{ }^{11}\right)$ and in the Nelson study resectability of diagnosed lung malignancies was $67.7 \%$ (ref. $^{12}$ ). This could be due to the fact that in our study also persons monitored for other lung diseases (COPD) were examined, and not only asymptomatic smokers as were included in above mentioned studies.

Our results are comparable with other studies. The false positivity of CT-controlled biopsy is usually very low, up to $0.2 \%$ (ref. ${ }^{13,14}$ ). False negativity varies between $6-54 \%$. Our results correspond with these data. Only $1 / 50$ punctures diagnosed as lung carcinoma was discordant with the final surgical specimen diagnosis. The sensitivity in our cohort was $81.7 \%$.

The proportion of PNOs occurring after biopsy is reported in the literature in the range of $12-45 \%$, while PNOs requiring drainage arise in around $2-15 \%$. 
According to the literature, cases of postpunction hemoptysis vary between $1.7-10.3 \%$ (ref. $^{3,15-18}$ ). Our results are in agreement with the reported numbers of both minor and severe complicating PNOs as well as the number of patients with hemoptysis.

\section{CONCLUSION}

Biopsy of peripheral lung lesions under CT control is an effective and safe method that will become increasingly important for diagnosis. Although a number of complications can be expected in about a third of patients, they are serious in a few percent only. The accuracy of this method in the diagnosis of small sized lung cancers is sufficient, we verified by biopsy under CT control $82 \%$ of LC. With the increasing number of detected pulmonary lesions in connection with planned lung cancer screening, there will be an increasing demand for quality material obtained by biopsies, that will be needed both for basic morphological verification as well as for biomarker testing necessary for targeted treatment of lung cancer. Therefore, we are convinced that the importance and number of indications of this method will increase in the future. Our aim is to contribute to a better awareness of what can be expected from biopsy of pulmonary nodules under CT control, both by indicating physicians as well as by performing radiologists.

Acknowledgement: Supported by the project (Ministry of Health) of the conceptual development of the research organization 00064203 (FN Motol).

Author contributions: MM, RP, LF: Manuscript writing, data analysis; DR, FCM, LC: Literature search and final approval; VC, RP: Data analysis and statistics.

Conflict of interest statement: The authors state that there are no conflicts of interest regarding the publication of this article.

\section{REFERENCES}

1. Barnett J, Belsey J, Tavare AN, Saini A, Patel A, Hayward M, Hare SS. Pre-surgical lung biopsy in management of solitary pulmonary nodules: a cost effectiveness analysis. J Med Econ 2019;22(12):1307-11.

2. Wu CC, Maher MM, Shepard JA. Complications of CT-guided percutaneous needle biopsy of the chest: prevention and management. AJR Am J Roentgenol 2011;196(6):W678-82.

3. Gupta S, Wallace MJ, Cardella JF, Kundu S, Miller DL, Rose SC. Society of Interventional Radiology Standards of Practice Committee. Quality improvement guidelines for percutaneous needle biopsy. J Vasc Interv Radiol 2010;21(7):969-75.
4. Tsai PC, Yeh YC, Hsu PK, Chen CK, Chou TY, Wu YC. CT-Guided Core Biopsy for Peripheral Sub-solid Pulmonary Nodules to Predict Predominant Histological and Aggressive Subtypes of Lung Adenocarcinoma. Ann Surg Oncol 2020; May 3 doi: 10.1245/s10434020-08511-9. Online ahead of print

5. Shen $\mathrm{H}$. Low-dose CT for lung cancer screening: opportunities and challenges. Front Med 2018;12(1):116-21.

6. Huang MD, Weng HH, Hsu SL, Hsu LS, Lin WM, Chen CW, Tsai YH. Accuracy and complications of CT-guided pulmonary core biopsy in small nodules: a single-center experience. Cancer Imaging 2019;23;19(1):51.

7. Dominguez-Konicky L, Karam AR, Furman MS, Grand DJ. CT-guided biopsy of pulmonary nodules $\leq 10 \mathrm{~mm}$ : Diagnostic yield based on nodules' lobar and segmental distribution. Clin Imaging 2020;66:7-9.

8. Marel M, Krejbich F, Stranska P. Bronchoscopic findings and their connection with other characteristics in 272 patients with lung cancer at the 1st Pulmonary Clinic, 1st Faculty of Medicine, Charles University and General Hospital Prague in the years 2004-2006. Stud Pneumol Phthiseol 2008;68(2):60-74.

9. Hsia DW, Jensen KW, Curran-Everett D, Musani Al. Diagnosis of lung nobles with peripheral/radial endobronchial ultrasound - guided transbronchial biopsy. J Bronchology Intern Pulmonol 2012;19(1):511.

10. Infante M, Cavuto S, Lutman FR, Brambilla G, Chiesa G, Ceresoli G, Passera E, Angeli E, Chiarenza M, Aranzulla G, Cariboni U, Errico V, Inzirillo F, Bottoni E, Voulaz E, Alloisio M, Destro A, ROncali M, Santoro A, Ravasi G; DANTE Study Group. A Randomized Study of Lung Cancer Screening with Spiral Computed Tomography. Three-year Results from the DANTE Trial. Am J Respir Crit Care Med 2009;180:445-53.

11. The National Lung Screening Trial Research Team. Reduced LungCancer Mortality with Low-Dose Computed Tomographic Screening. N Engl J Med 2011;365:395-409.

12. Walter JE, Heuvelmans MA, de Bock GH, Yousaf-Khan U, Groen HJM, van der Aalst CM, Nackaerts K, van Ooijen PMA, de Koning $H J$, Vliegenthart R, Oudkerk M. Relationship between the number of new nodules and lung cancer probability in incidence screening rounds of CT lung cancer screening: The NELSON study. Lung Cancer 2018;125:103-8.

13. Winokur RS, Pua BB, Sullivan BW, Madoff DC. Percutaneous lung biopsy: technique, efficacy, and complications. Semin Intervent Radiol 2013;30(2):121-7.

14. Yun S, Kang H, Park S, Kim BS, Park JG, Jung MJ. Diagnostic accuracy and complications of CT-guided core needle lung biopsy of solid and part-solid lesions. Br J Radiol 2018;91(1088):20170946.

15. Heerink WJ, de Bock GH, de Jonge GJ, Groen HJ, Vliegenthart R, Oudkerk M: Complication rates of $\mathrm{CT}$-guided transthoracic lung biopsy: meta-analysis. Eur Radiol 2017;27(1):138-48.

16. Yoon SH, Park CM, Lee KH, Lim KY, Suh YJ, Im DJ, Hur J, Han DH, Kang MJ, Choo JY, Kim C, Kim Jl, Hong H. Analysis of Complications of Percutaneous Transthoracic Needle Biopsy Using CT-Guidance Modalities In a Multicenter Cohort of 10568 Biopsies. Korean J Radiol 2019;20(2):323-31.

17. Tongbai T, McDermott S, Kiranantawat N, Muse VV, Wu C, Omalley Shepard JA, Gilman MD. Non-Diagnostic CT-Guided Percutaneous Needle Biopsy of the Lung: Predictive Factors and Final Diagnoses. Korean J Radiol 2019;20(11):1515-26.

18. Drumm O, Joyce EA, de Blacam C, Gleeson T, Kavanagh J, McCarthy E, McDermott R, Beddy P. CT-guided Lung Biopsy: Effect of Biopsyside Down Position on Pneumothorax and Chest Tube Placement. Radiology 2019;292(1):190-6. 